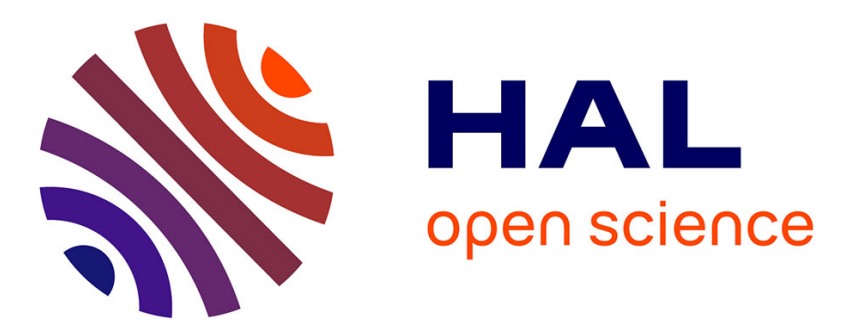

\title{
Sur la possibilité d'étudier l'aimantation nucléaire de longue persistance et sa relaxation par des méthodes simples
}

\author{
A. Kastler
}

\section{- To cite this version:}

A. Kastler. Sur la possibilité d'étudier l'aimantation nucléaire de longue persistance et sa relaxation par des méthodes simples. J. Phys. Radium, 1951, 12 (4), pp.567-569. 10.1051/jphysrad:01951001204056701 . jpa-00234425

\section{HAL Id: jpa-00234425 https://hal.science/jpa-00234425}

Submitted on 1 Jan 1951

HAL is a multi-disciplinary open access archive for the deposit and dissemination of scientific research documents, whether they are published or not. The documents may come from teaching and research institutions in France or abroad, or from public or private research centers.
L'archive ouverte pluridisciplinaire HAL, est destinée au dépôt et à la diffusion de documents scientifiques de niveau recherche, publiés ou non, émanant des établissements d'enseignement et de recherche français ou étrangers, des laboratoires publics ou privés. 
enregistrées pour tous les types de diaphragmes et la figure \& représente les variations (normalisées) de l'éclairement central. L'efficacité de $L_{0}$ au foyer s'explique suffisamment par cette variation, mais non celle de $\mathrm{C}_{0}$ avec fente. Pour l'expliquer on peut déterminer et comparer entre elles, d'après la figure 5,

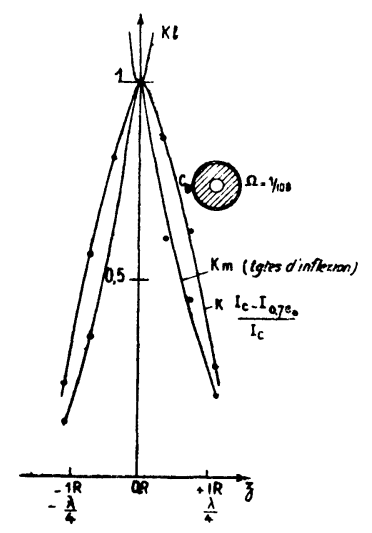

Fig: 5 .

les variations (normalisées) des différentes grandeurs auxquelles l'œil pourrait être sensible, largeur du pic central, pente d'inflexion de ce pic, contraste $\frac{I_{c}-I_{x}}{I_{c}}$ entre le centre et l'“ épaulement " voisin; c'est cette dernière grandeur qui semble intervenir. La figure 3

\section{SUR LA POSSIBILITE \\ D'ÉTUDIER L'AIMANTATION NUGLEAIRE DE LONGUE PERSISTANGE \\ ET SA RELAXATION PAR DES METHODES SIMPLES}

Par A. KASTLER, Laboratoire de Physique de l'École Normale Supérieure.

Sommaire. - La grande durée de relaxation de certaines aimantations nucléaires permet d'envisager l'étude de 
ces phénomènes et de leurs mécanismes de relaxation en dehors du champ inducteur. Une évaluation numérique montre que la grandeur du paramagnétisme nucléaire est suffisante, à basse température surtout, pour être mesurée par les méthodes classiques du magnétomètre ou de la bobine tournante fournissant une f.é.m. d'induction.

A la suite d'une suggestion de J. Dorfmann [1] les physiciens russes Lasarew et Schubnikow [2] ont réussi dès $I^{3} 7$ à mesurer le paramagnétisme nucléaire du proton dans l'hydrogène solide entre 4,22 et $1,76^{\circ} \mathrm{K}$ par la méthode classique de la balance dans un champ magnétique non uniforme.

D'autre part les expériences de résonance paramagnétique nucléaire ont mis en évidence l'orientation des noyaux atomiques dans les champs magnétiques [3] et ont permis de mesurer leurs durées de relaxation, c'est-à-dire le temps au bout duquel les noyaux se redistribuent au hasard après suppression du champ. Ce temps peut, dans certain cas, avec des échantillons de très grande pureté, atteindre des valeurs de plusieurs minutes [4]. Lorsqu'on éloigne l'échantillon nucléaire "aimanté " du champ, il conserve pendant un certain temps son aimantation, qui ne décroît que lentement. On peut dire que les noyaux, en quittant le champ, subissent la désaimantation adiabatique qui les porte à une " température de spin nucléaire " extrêmement faible et qu'ils ne se réchauffent que lentement au contact du réseau [5].

Quel est l'ordre de grandeur de l'aimantation persistante qu'on peut ainsi créer ? Elle est donnée par la loi de Boltzmann où $T$ représente la température de l'échantillon placé dans le champ $H$. Pour lé cas simple où le spin nucléaire est $i=\frac{\mathrm{I}}{2}$ et où les noyaux ne possèdent que deux orientations opposées dans le champ : $m_{i}=-\frac{1}{2}$ et $m_{i}=+\frac{1}{2}$ de différence d'énergie $\Delta E=2 \mu H$ ( $\mu$ étant le moment nucléaire lié au spin $i$ ), les populations des deux niveau sont donnés par

$$
\frac{n_{-}}{n_{+}}=\mathrm{e}^{-\frac{\Delta E}{k T}} \quad \text { ou } \quad \frac{\Delta n}{n}=\frac{\Delta E}{k T} .
$$

Dans un champ de 5 o ooo gauss les $\Delta E$ sont de l'ordre de $\mathrm{i}$ oo à $200 \mathrm{Mc}: \mathrm{s}$. Prenons la valeur moyenne

$$
\text { I } 50 \mathrm{Me}: \mathrm{s}=5 \cdot 1 \mathrm{O}^{-3} \mathrm{~cm}^{-1} \text {. }
$$

L'énergie $k T$ correspond à $200 \mathrm{~cm}^{-1}$ à la température ordinaire $\left(300^{\circ} \mathrm{K}\right)$, à I $\mathrm{cm}^{-1}$ seulement à la température de $\mathrm{I}, 5^{\circ} \mathrm{K}$ réalisable avec l'hélium liquide. On a donc

$$
\frac{\Delta n}{n} \sim 2,5 \cdot 10^{-5} \text { à } 300^{\circ} \mathrm{K} \text { ou } \sim 5 \cdot 10^{-3} \text { à } \mathrm{I}, 5^{\circ} \mathrm{K} .
$$

La substance solide sera par exemple un bloc de Li F qui contient environ $0,5.10^{23}$ noyaux par centimètre cube dont le moment magnétique est de l'ordre de

$$
\mu_{N} \approx \mathrm{IO}^{-3} \mu_{B} \sim \mathrm{IO}^{-23} \mathrm{erg} \cdot \text { gauss }^{-1} \text {. }
$$

L'intensité d'aimantation (moment magnétique par centimètre cube) est donc de l'ordre de

$$
I \sim 5 \cdot 10^{-3} \cdot 0,5 \cdot 10^{23} \cdot 10^{-23}=2,5 \cdot 10^{-3} \text { à } \mathrm{I}, 5^{\circ} \mathrm{K},
$$

ou $\mathrm{IO}^{-5}$ à la température ordinaire.
En aimantant deux blocs d'échantillon pendant quelques minutes dans un champ de 50 ooo gauss, en les retirant rapidement du champ et en les rapprochant, on aura dans l' "entrefer" entre les deux blocs, un champ initial de l'ordre de $H_{n} \sim 2 \pi I$, soit environ $10^{-2}$ gauss à $1,5^{\circ} \mathrm{K}$ ou $5.10^{-5}$ gauss à température ordinaire (voir Appendice).

Un magnétomètre ayant comme champ directeur le champ terrestre $(H=0,2$ gauss) permet de déceler un champ perpendiculaire de 5. 1 $0^{-6}$ gauss [6]. Muni d'un couple astatique et d'un champ directeur dix fois plus faible que le champ terrestre, un tel appareil permet donc de déceler sans difficulté des champs de $1^{-6}$ gauss [7], soit encore 50 fois plus faibles que les champs produits par les aimantations nucléaires à la température ordinaire. (Toutefois, à la température ordinaire, le diamagnétisme induit dans l'échantillon par le champ directeur et par l'aimant du magnétomètre est du même ordre de grandeur que le magnétisme nucléaire et il y a lieu d'en tenir compte. Cette difficulté. n'existe pas quand on emploie la méthode de la bobine tournante).

On pourra donc aisément déceler par des mesures magnétostatiques les aimantations nucléaires persistantes, étudier leur décroissance en fonction du temps et recueillir des renseignements utiles sur les mécanismes de relaxation dans des champs nuls.

On peut employer une méthode d'induction basse fréquence en faisant tourner autour des aimants nucléaires une petite bobine dont on amplifie la f.é.m. induite, etc.

Notons aussi qu'un échantillon soumis à un champ magnétique tournant devient le siège d'un couple qui peut devenir important lorsque la période de rotation du champ est de l'ordre de la durée de relaxation de l'échantillon (de l'ordre de $I . H$ pour $\mathrm{I} \mathrm{cm}^{3}$, soit environ 0,5 erg-radian dans un champ de 50 ooo gauss à la température ordinaire ( 100 ergradian avec $\mathrm{He}$ liquide!).

On peut se demander ce qui se passe si dans le champ magnétisant ou en dehors de ce champ on retourne l'échantillon aimanté bout. pour bout ? Les noyaux tournent-ils avec le morceau de matière ou conservent-ils leur orientation dans l'espace ? La première alternative nous paraît probable, au moins en dehors du champ. S'il en est ainsi la rotation mécanique de l'échantillon doit produire des effets d'induction faciles à déceler avec des amplificateurs basse fréquence, et si l'échantillon est sphérique et isotrope les effets de diamagnétisme n'interviennent pas.

Appendice. - Le champ $H$ au centre de symétrie de l'" entrefer " entre deux blocs cylindriques alignés est donné par

$$
H=4 \pi\left(\cos \alpha_{2}-\cos \alpha_{1}\right) I,
$$

où $\alpha_{2}$ et $\alpha_{1}$ représentent les demi-angles sous lesquels sont vu les faces circulaires externes et internes.

Pour deux cylindres de $\mathrm{I} \mathrm{cm}^{3}$ de section et $\mathrm{I} \mathrm{cm}$ de longueur, laissant entre eux un "entrefer" de $5 \mathrm{~mm}$ d'épaisseur :

$\alpha_{1}=66^{\circ}, \quad \cos \alpha_{1}=0,406, \quad \alpha_{2}=25^{\circ}, \quad \cos \alpha_{2}=0,906 ;$
$H \approx 2 \pi I$.

Dans la méthode d'induction on a intérêt à faire 
tourner un cerceau autour d'un échantillon sphérique. La variation de flux par demi-tour est de l'ordre de

$$
\Delta \Phi=2 \frac{4 \pi}{3} I \pi r^{2}
$$

soit pour $r=\mathrm{I}$ cm et $I \sim \mathrm{IO}^{-5}$ de 2,5. IO $\mathrm{I}^{-3}$ Maxwell.

$\mathrm{Si}$ le cerceau porte $\mathrm{I}^{3}$ spires et tourne à raison de $50 \mathrm{t}: \mathrm{s}$ :

$$
\mathrm{e}=1 \mathrm{IO}^{-8} \cdot 10^{3} \frac{2,5 \cdot 10^{-3}}{10^{-2}}=2,5 \cdot 10^{-6} \mathrm{~V}
$$

valeur facile à amplifier par un procédé classique.

[1] Dorfmann J. - Physik. Zeitschr. Sowjet Union, 1935. 7, 126.

[2[ Lasarew B. G. et Schubnikow L. W., Ibid., i 937 , 11, 445 .

[3] Soutif M. - J. Physique Rad., r 949, 10, 6r D.

[4] Pound R. V.- Phys. Rev., г 95 г , 81, i 56, et r $950,79,685$.

[5] Purcell E. M. - Congrès Radiofréquences, Amsterdam, 1950.

[6] Bruhat G. - Cours d'Électricité, Chapitre Magnétisme, $\S 101$ et 249.

[7] Forrer R. - J. Physique Rad., i $929,10,247,252 .-$ Thellier. - Ann. Inst. Phys. Globe, Paris, I 938, XVI, 157, 167 .

Manuscrit reçu le 24 février $195 \mathrm{I}$. 